\title{
Shape Optimisation of Composite Corrugated Morphing Skins
}

\author{
Anna Ermakova ${ }^{1}$, Iman Dayyani ${ }^{2}$ \\ ${ }^{1}$ Faculty of Engineering and Computing, Coventry University, Coventry, CV1 5FB, UK, ermakova@uni.coventry.ac.uk \\ ${ }^{2}$ Corresponding author; Centre for Structures, Assembly and Intelligent Automation, \\ Cranfield University, MK43 OAL, UK, I.Dayyani@Cranfield.ac.uk
}

\begin{abstract}
One of the challenging parts of the morphing wing is the anisotropic skin, which must be flexible enough to allow the wing to change its shape and at the same time being stiff enough to withstand the aerodynamic loads. Composite corrugated skins have exceedingly anisotropic behaviour as they are stiff along the corrugation direction but flexible in transverse direction. Hence, elastomeric coated composite corrugated panels have been proposed as a candidate for application in morphing wings. This paper presents the shape optimisation of the corrugation with respect to better performance of the morphing skin and manufacturing constraints. The shape of the skin is optimised by minimising the in-plane stiffness and weight of the skin and maximising its flexural out-of-plane stiffness. The objective functions were obtained from homogenised model that depends on geometric and mechanical properties of the coated corrugated panel by means of finite element method for thin beams. A few methods of optimisation were considered: aggregated and genetic algorithm methods as representative of two major categories of multi-objective solving methods. A number of different approaches are proposed in order to solve the problem, such as corrugated skin with and without elastomer coating. The advantages of the new optimised shape of the corrugated skin over the typical shapes are discussed.
\end{abstract}

\section{Keywords}

Anisotropy, Elasticity, Computational modelling, Finite element analysis (FEA) 


\section{Introduction}

The main technology that is used nowadays to control the induced aerodynamic forces and moments on the aerofoil is through changing its camber by means of deployed flaps or slats. The amount of control which is gained is significant despite the fact that only small portion of the aerofoil is able to rotate. Due to simplicity and efficiency of this technology it now has a wide range of applications within the industry [1]. However, the drawbacks of using flaps should be mentioned here as well. Discontinuous shape of aerofoil leads to increase of pressure and flow separation that causes the large rise in drag [2].

Therefore, a lot of research into morphing wing technology has been made over the decade since its smooth and continuous camber change provides improvements in aerodynamic efficiency and expands the flight envelope $[3,4,5]$. Moreover, the complex mechanism of flaps and slats increases the weight as well as assembly and operational costs.

A lot of different concepts of morphing wing mechanisms were introduced. Several examples were shown in the literature; Woods, Fincham, and Friswell [6]. It is highlighted in the literature that morphing concepts are usually very complex and difficult to implement. Thus the need to have a simple and reliable design of morphing wing is very important. This will result in cost reduction, minimize the number of components and finally weight of the structure [7]. In light of all these criteria; the Fish Bone Active Camber (FishBAC) concept was introduced by Woods and Friswell [8] inspired by nature. The design is shown in Fig. 1. The components of the architecture are thin chord - the spine, which provides wise bending, and stringers that connect the spine with an elastomeric matrix composite (EMC) skin. The bending moment is provided by means of actuator, which is located in non-flexible leading edge of the aerofoil.

Tests of this new design of morphing aerofoil structure have demonstrated the increase of out-of-plane stiffness and decrease of surface skin buckling. Also it was found that this concept improves the aerodynamic efficiency compared to conventional concept flaps. Moreover, this structure provides larger available deflection of continuous surface. 
In paper by Dayyani et al. [9] it was considered to replace the EMC skin with composite corrugated skin coated with elastomer. Composite corrugated panels are highly anisotropic. This means that corrugated core is stiff along the corrugation direction and flexible in the transverse direction [10]. It is a main reason of using this skin in morphing application as skin must be stiff enough to withstand the aerodynamic loads and soft enough to change the shape of the wing.

In paper by Dayyani, Ziaei-Rad, and Friswell [11] the equivalent structural models were proposed that sustains the dependence on parameters of geometry of the corrugated skin. Two models represent two analytical solutions in order to calculate the equivalent bending and tensile of the skin in the transverse and longitudinal directions through Finite Element Method analysis, which was previously implemented in paper by Gilchrist et al. [12].

Different configurations of the corrugated core can be used [13]. Therefore, in order to find the best one among three typical shapes the research was carried out by Dayyani et al. [14], optimising the structure of the skin in terms of two objectives: the ratio of tensile stiffness to flexural stiffness and the mass. The corrugated core with and without elastomer coating was investigated.

This work contains a further research into the problem and suggests solution in order to improve the efficiency of morphing wing by introducing a new optimised shape of corrugated skin. The shape of the coated composite corrugated panels are optimised to minimize the in-plane stiffness and the weight of the skin and to maximize the flexural out-of-plane stiffness of the corrugated skin. These objective functions were calculated by use of an equivalent finite element code for thin beams. The gradient-based aggregate method is selected to solve the optimisation problem. The advantages of the optimised-shape of corrugated skin over former skins for the camber morphing structure are discussed. 


\section{Problem Definition}

\subsection{Equivalent structural properties of the coated corrugated core.}

FEM was generated in MATLAB for thin beam elements to calculate the equivalent tensile stiffness, flexural stiffness and the mass of a corrugated core which contains four unit cells. A unit cell of corrugation with arbitrary shape is shown in the Fig. 3.

\subsubsection{FE Analysis and material properties}

The indicated area ' $A B C D$ ' is the area of interest that represents the corrugation section. Since the shape of the corrugation ' $A C$ ' is unknown, the FE code is created in such a way that for any number of nodes, which lay in this area of interest, the new shape will be built and then the equivalent structural stiffness and the mass will be calculated. Coordinates of those nodes are inputs of the FE code. It is important to add here, that separate nodes ' $A$ ' and ' $C$ ' are considered as existing nodes by default, i.e. not included to the area of interest.

Parameters $a, h, l$ and the width of the panel $b$ which define the geometry of the corrugated structure are considered as fixed values, as shown in Table 1 . The thickness of the corrugated core $t_{c}$ and elastomer coating $t_{e}$ are included as design parameters. Moreover, the material properties such as: $\rho_{c}, \rho_{e}, E_{c}$ and $E_{e}$ which are the density of corrugated core and elastomer coating as well as their Young's modulus respectively, are presented in Table 1. More details of the FE modelling can be found in [9].

As can be noticed from the Fig. 3, there are some areas in the unit cell structure, where elastomer coating overlaps the corrugated core. Working under the assumption that these two materials are well adhered together, and considering that the ratio of Young's modulus of elastomer coating to Young's modulus of composite corrugated core is very small, the elastomer coating was neglected in these areas [11].

\subsubsection{Sketch of the corrugated structure}

The corrugated structure was discretised into small 2D beam elements and nodes. The 2D beam model is justified as the corrugated panel is not subjected to any load or deformation in the third direction. Since number of elements depends on number of nodes that corrugation 'AC' contains as shown in Fig. 3, a set of independent nodes for structure was established as shown in Fig. 4, where $n$ is the number of nodes on the corrugation profile 'AC'. Hence, the total number of nodes of the structure is equal to $(20+8 n)$. Three 
degrees of freedom $U_{x}, U_{y}$ and $\theta_{z}$ in the global coordinate system were considered at each node, therefore the degrees of freedom of the entire structure is $3 \cdot(20+8 n)$, and number of elements is equal to $(18+8$. $(n+1))$.

\subsubsection{Connectivity of Nodes}

It was observed using MATLAB, that in case if length of element is less than $0.001 \mathrm{~mm}$, there is an error which occurs calculating the displacement, warning that the matrix is close to singular and the result can be not accurate enough. Since this length is so small it will not give a visible change of the shape of corrugation anyway and can be considered that these two nodes, which create this element, have the same coordinates.

Therefore, a number of checks were implemented in the beginning of the code to ensure that all nodes are far enough from each other, so the error no longer occurs. Such as If the difference between coordinates of two neighbour nodes is less than $0.001 \mathrm{~mm}$, i.e. $\left|x_{1}-x_{2}\right|<0.001$ and $\left|y_{1}-y_{2}\right|<0.001$, then two nodes were considered as identical nodes, i.e. $x_{1}=x_{2}$ and $y_{1}=y_{2}$ and the repetitive node was ignored.

Another important check, if neglected can lead to significant change of the structure is that: If at least one of the nodes of corrugation profile ' $A C$ ' is placed on the line segment ' $B C$ ' or ' $A D$ ' of the domain boundaries, then the elastomer coating will no longer connect to the node ' $C$ ' or node ' $A$ ' respectively. For instance, if the corrugated profile ' $A C$ ' has only one node and that node will be selected by the optimisation identical to node ' $B$ ', then corrugation will have rectangular shape ' $A B C$ ', in this case the elastomer coating will be connected to node ' $B$ ' instead of node ' $C$ '.

\subsubsection{Validation of the structural code}

The FE code was generated to calculate equivalent tensile and bending stiffness of the structure. The results for both were verified using ABAQUS to ensure the validity of the structural analysis. Identical structure of coated corrugated core was modelled in ABAQUS using beam elements with a fine mesh, identical boundary conditions and applied external forces. The fixed geometric parameters and material properties were set as per Table 2. Prescribed boundary conditions for both cases of loadings are presented in Table 3. A variety of nodes numbers, node coordinates, thickness of materials and forces values were investigated in the comparison. 
Figure 5 demonstrates the undeformed and deformed shapes of the structure subjected to both tensile and bending forces for case when $n=2$, coordinate of the node are $x_{1}=2.3, y_{1}=3.4, x_{2}=7.7, y_{2}=6.2$, thickness of the core and elastomer are $t_{c}=0.27(\mathrm{~mm}), t_{e}=0.55(\mathrm{~mm})$. The bending force is $F_{\text {bend }}=$ $-0.01(N)$ applied to a node $18+8 n$ (Fig.4), tensile force $F_{\text {tens }}=5(N)$ applied to nodes $18+8 n$ and $19+8 n$

Table 4 shows that for both loading cases the difference of the results obtained in both software is within 1.11\%. The cubic shape functions of the beam element interpolate the deformation precisely therefore provides highly accurate results. Convergence analysis was conducted by changing the size of the mesh, practically the appropriate size of the mesh was established, which compromises between high accuracy of results and computational time [9].

\subsection{Multi-objective optimisation}

The multi-objective optimisation was performed in order to minimize three objectives: the equivalent tensile stiffness $E A_{e q}$, reciprocal of the equivalent flexural stiffness $1 / E I_{e q}$, and mass of the corrugated core. Each of these three objectives has its own impact on the performance of the aircraft as a whole system. The equivalent tensile stiffness is related to the actuator force, which is required, therefore it should be minimized to minimize the energy consumption. The equivalent flexural stiffness represents the out-of-plane deformation of the skin, which occurs under the aerodynamic pressure loading, hence it should be maximized to increase the deformation resistance. Mass of the skin is a component of the total mass of the aircraft thus it has to be minimized as well. Optimising these three objectives results in further efficiency of the aircraft.

Since the order of magnitude of these three objectives which are to minimize, is different; for instance for $E A_{e q}$ it is usually $10^{3}$, where as for $1 / E I_{e q}$ it is $10^{-4}$, therefore all the parameters were normalized, to ensure the best performance of the optimisation scheme as:

$$
x_{n}(i)=\frac{x(i)-\left(\frac{x_{u}(i)+x_{l}(i)}{2}\right)}{\left(\frac{x_{u}(i)+x_{l}(i)}{2}\right)}, \quad i=1,2,3 \ldots, n
$$

where $x_{n}$-normalized vector of parameters, $x$ is a vector of parameters, $x_{u}$ and $x_{l}$ represent upper and lower bound vectors, $i$ - number of parameters, which depends on number of nodes line AC contains. The objective 
functions were also normalized as $f_{n}(x)=f(x) / f(\bar{x})$, where $\bar{x}=\left(x_{u}+x_{l}\right) / 2$ - average vector of upper bound and lower bound values [9].

\subsubsection{Boundaries and Constraints}

Selection of the upper and lower bounds for the thickness of corrugated core and elastomer coating was based on practical consideration, as with high ratio of the thickness of the corrugated core to the length of unit cell, mechanism of deformation changes, providing a panel which is very stiff. Thus the upper bound for the thickness of corrugated core was set to $l / 10(\mathrm{~mm})$, the same upper bound was set for the thickness of elastomer coating. The lower bounds were defined by the availability of the selected material as $l / 100(\mathrm{~mm})$ for both corrugated core and elastomer coating.

To represent results of optimisation, Pareto surfaces were plotted for each optimisation case as shown in Fig. 6. Moreover, for each configuration the best compromise point was selected using following technique. First, coordinates of an ideal reference point were identified by selecting the minimum values of each objective from the set of solutions for given optimisation:

$$
P_{\text {ref }}=\left(\left(E A_{e q}\right)_{\text {min }},\left(\frac{1}{E I_{e q}}\right)_{\text {min }},(\operatorname{mass})_{\min }\right)
$$

After that the point with minimum distance from the reference point was found from that set of normalized objective functions, which represents the best compromise point.

In this work in order to solve multi-objective problem the aggregate method (AG) from classical methods and the Genetic Algorithm (GA) from evolutionary methods were selected to compare their performance and then choose better one to perform the optimisation.

\subsubsection{Aggregate method}

There are some application areas where this method is limited to be used, specifically if objectives and constraints functions are both not continuous. However, it does not restrict the use of this method for current optimisation problem, as the continuous analytical solutions have been proposed [15]. Using this method 
three objectives were combined into one single objective function. This approach is to minimize a linear combination of positively weighted objectives:

$$
f(x)=w_{1} \cdot f_{1}(x)+w_{2} \cdot f_{2}(x)+w_{3} \cdot f_{3}(x)
$$

In MATLAB this optimisation method can be performed by using command 'fmincon' - gradient based method [16]. Weighting coefficients should be positive and show the importance of particular objective in relation to other objectives. The sum of three weighting coefficients is assumed to be equal to one. Since the solution of optimisation problem can vary as weights change and considering the fact that reducing the interval of weight coefficients leads to a larger number of points of Pareto curve, hence more accurate solution of optimisation can be found, therefore the weights were selected to vary from 0.01 to 0.98 with increments of 0.01

Optimisations with different number of nodes were carried out to examine the shapes provided by the current optimisation method. It was revealed that despite the slight shape difference the similar pattern of corrugation shapes can be observed as shown in Fig. 7. This demonstrates the optimisation shape convergence, which is very important. Considering the computation time due to number of optimisation parameters, since the pattern for all examined nodes (1-10) consists of two-three straight lines, which can be plotted with maximum two nodes, it was decided to continue the research considering only two nodes between nodes ' $A$ ' and ' $C$ '.

\subsubsection{Genetic algorithm method}

A single solution for multi-objective problems, which optimise all objectives at the same time, does not exist due to its conflicting nature. A process of improving at least one objective function without making remaining objectives worse is known as Pareto improvement. If further improvement is not possible the set of individuals is called Pareto optimal. There are plenty different techniques, which based on selecting the Pareto optimal set. For the current work a Pareto based GA technique known as 'gaoptimset', was chosen in MATLAB to solve the optimisation problem. According to paper by Dayyani, Friswell [17] all settings for the optimisation were set as default except only two: the crossover function, which was set to 0.3 , and the population size, set to 200. Since GA randomly generates the initial population set of individuals [18], for the identical setting parameters the optimised shape was too different. In other words, the randomness of GA method did not 
provide a certain pattern of the shape. In addition, the shapes obtained using this method were less smooth compared to results obtained with aggregate method. Moreover, GA algorithm gives worse results of objective values of compromise point for both cases increasing all of them: $E A_{e q}$ by $5-12 \%, 1 / E I_{e q}$ by $4-17 \%$ and $\operatorname{mass}$ by $13-14 \%$ compared to results of aggregate method. Therefore, it was decided to continue the optimisation of the problem using aggregate optimisation method for two nodes following different approaches listed in section 2.3 .

\subsection{Different approaches to the problem}

In order to find an optimal shape, different boundaries for node coordinates were implemented, which are as follows:

1) Optimisation domain without boundary conditions for nodes within the area ' $A B C D$ ': $0 \leq x \leq l$ and $0 \leq y \leq h$. This method provides freedom to optimiser to build any profile of the corrugation.

2) Optimisation domain with boundary for the $2^{\text {nd }}$ node, in order to secure an ascending curve of corrugation to ease the manufacturing process and limitations, i.e.: $x_{1}<x_{2}<l$ and $y_{1}<y_{2}<h$.

3) Boundary only for the $\mathrm{X}$ coordinates: $x_{1}<x_{2}<l$, to investigate the effect of horizontal degrees of freedom.

4) Boundary only for the $Y$ coordinates: $y_{1}<y_{2}<h$, to investigate the effect of vertical degrees of freedom.

5) 4 subdomains method, which enforces the code to build all possible types of corrugation shapes. Dividing the optimisation domain into four subdomains as shown in the Fig. 8, optimisation was run 16 times for all possible combinations of two nodes locations. Then the best result was selected as the best compromise point.

All of the above methods were considered for the following cases:

1) With and without elastomer coating, to assess the performance of the coated and uncoated skins.

2) With and without fixed thickness of materials. In the case of fixed thickness of materials, the optimisation process will focus only on the optimisation of the corrugation shape with uniform thickness. The thickness parameters for the uniform shape optimisations were considered as:

- $\quad t_{c}=0.25 \mathrm{~mm}$ and $t_{e}=0.55 \mathrm{~mm}$ with elastomer coating 
- $t_{c}=0.5 \mathrm{~mm}$ without elastomer coating

\section{Results and discussions}

\subsection{Case of coated corrugated core}

\subsubsection{Unfixed thickness of materials}

First, the case of coated corrugated skin was investigated. The results obtained for the case of unfixed thickness of materials are shown in Fig. 9, where one half of unit cell is presented. Lines representing different materials, are given different thickness so as to visualise the ratio of thicknesses of composite core to elastomer coating.

Similar pattern of corrugations can be noticed, however the shape obtained by means of four subdomains method differs from the rest. It should be mentioned here that although the four subdomains approach results in a different configuration, yet it is a potential configuration among the optimised shapes. The reason is due to the different mechanism of deformation, i.e. spring effect in this pattern. The spring effect increases the ratio out of plane stiffness to in plane stiffness for increase of mass. Comparative analysis of objective values and thickness of materials is presented in Fig. 10.

Figure 10 shows that in fact the best compromise point among all the results is for the four subdomains method. The ratio between thicknesses of composite material and elastomer coating varies from 0.44 to 0.56 , whereas for the best case is equal to 0.56 . It also can be noted that the value of thickness of elastomer coating just slightly changes among the results from 0.54 to $0.56 \mathrm{~mm}$.

\subsubsection{Fixed thickness of materials}

Then the case of coated corrugated core with fixed thickness of materials was examined, corrugation shapes are shown in Fig. 11.

The pattern of the obtained shapes for this approach shows that the optimisation is mainly minimizing mass of the structure and hence reveals trapezoidal patterns but with higher angle of corrugation. In fact, the higher 
angle of corrugation results in higher out of plane stiffness to resist the aerodynamic pressure and buckling forces as well as lower in plane stiffness to reduce actuation energy required. This is why it differs from the pattern obtained for the previous case of unfixed thickness.

Considering the comparative analysis of objective values shown in Fig. 12, the best compromise point for this case is again obtained by means of four subdomains method. Moreover, it can be concluded that fluctuation range of objective values in case of fixed thickness is $2-6 \%$ times narrower for $1 / E I_{e q}$ and mass respectively, however, $4 \%$ more wide for $E A_{e q}$ in contrast to the case of unfixed thickness.

\subsubsection{Comparison of unfixed and fixed thickness approaches for the case of coated corrugated core}

The best shape obtained within the case of coated corrugated core was selected as the best compromise point among all the results, which is the shape obtained by means of four subdomains method with unfixed thickness as shown in Fig. 13. It also should be mentioned here, that all of the results obtained in case of unfixed thickness are better compared to the results for the case with fixed thickness of materials. Therefore, it can be concluded that constant thickness of materials did not improve the objective values, especially for reducing the mass of structure.

This shows that the corrugation has a global trapezoidal shape but with a local spring component in the middle, the size of which is small ( $12 \%$ of the cell height) due to minimising the mass of the structure. However, this may lead to complexity of manufacturing process, therefore the second compromise point was selected as the decision making corrugation shape. This configuration was obtained with the approach of boundaries for $\mathrm{X}$ coordinates. Table 5 presents the objective values, thickness of materials and weights distribution for this configuration.

The ratio of $E A_{e q} / E I_{e q}=0.04319$ for this configuration is $0.4 \%$ higher than for the first compromise point with local spring component. Weighting coefficient values indicate that all three objectives were involved in the process of shape optimisation. Comparing the new optimised corrugation shape to existing trapezoidal corrugated shape in literature [9], the thickness ratio in the corrugated skin was increased to 2.12 times, which 
will lead to better characteristics of the skin. The shape optimised structure of the coated corrugated skin is presented in the Fig. 14.

In order to examine the morphing design consideration of the optimised coated corrugated skin, the structure was simulated in ABAQUS comprised of 10 unit cells. Different loading conditions were considered to ensure the proper performance of skin under different representative conditions that the airfoil would experience. A range of bending forces $(0-6) \cdot 10^{-3} N$ and a range of tensile forces $0-45 N$ were investigated. The simulations for following forces are presented: applied bending force $\mathrm{F}_{\text {bend }}=-0.006 \mathrm{~N}$, tensile force $\mathrm{F}_{\text {tens }}=40 \mathrm{~N}$. Distributed pressure of $500 \mathrm{~Pa}$ was considered for simulation of optimised skin under aerodynamic loads, which is the maximum pressure for NACA 0012, subjected to $30 \mathrm{~m} / \mathrm{s}$ airflow [9]. Boundary conditions for first two cases are the same as given in Table 3, whereas for the last case, the lower surface is considered fixed. The results are presented in Fig. 15.

From the Fig. 15a the local buckling of the corrugated skin can be observed as wrinkling of elastomer coating, which is caused by application of bending force. However, the results for simulation of skin under the aerodynamic load (Fig. 15c) do not show any wrinkling of elastomer. In manufacturing process buckling problem can be avoided by applying pre-stressed elastomeric coating as suggested in [9]. The ratio of force over displacement in case of bending case is equal to $0.0002 \mathrm{~N} / \mathrm{mm}$, for the case of tensile mode $0.63 \mathrm{~N} / \mathrm{mm}$.

\subsection{Case of uncoated corrugated core}

\subsubsection{Unfixed thickness of materials}

The results obtained for the case of unfixed thickness of materials is shown in Fig. 16.

Similarly to the case of coated corrugated core, the optimised shape by means of four subdomains approach is different from the rest.

Comparative analysis of objective values and thickness of materials are presented in Fig. 17. The best 
compromise point among all the results represents the results obtained by means of four subdomains method.

The results show that the thickness of corrugated core is increased twice in the optimisation process in contrast to elastomer coated corrugated core.

\subsubsection{Fixed thickness of materials}

Uncoated corrugated core with fixed thickness of materials was examined, optimised corrugation shapes are shown in Fig. 18.

Similarly to the case of coated core, optimisation results highlight a trapezoidal shape with high angle of corrugation.

Comparative analysis of objective values shown in Fig. 19 shows that the best compromise point for this case is again obtained by means of four subdomains method. Moreover, it can be concluded that fluctuation range of mass and $1 / E I_{e q}$ objective values is $1 \%$ and $26 \%$ narrower respectively, in compare to the case of unfixed thickness. In case of in plane stiffness i.e. $E A_{e q}$ it is $2 \%$ wider.

\subsubsection{Comparison of unfixed and fixed thickness approaches for the case of uncoated corrugated core}

The best optimised shape for the case of uncoated corrugated core was selected as the best compromise point among all the results, Figure 20 shows the best compromise optimised shape, which corresponds to four subdomains approach with constant thickness. This implies that constant thickness of materials leads to better values of objectives.

Objective values, thickness of materials and weighting coefficient distribution are given in Table 6.

The ratio of $E A_{e q} / E I_{e q}=0.095$ for the current case. The weights values indicate that second objective was almost neglected during optimisation.

The optimised structure of the uncoated corrugated skin is presented in the Fig. 21. 
Since application of the morphing wing requires the minimum tensile stiffness and bending flexibility as well as smooth surface of the skin for better aerodynamic performance, the configuration of uncoated corrugated core is not feasible for this application, however may be considered in other cases.

Similarly, to the case of coated skin, to examine the mechanism of deformation of the optimised coated skin, the structure was simulated in ABAQUS that contains 10 unit cells. Different load conditions were examined as was described in section 3.1.3. For visual presentation the following forces were applied: bending force $F_{\text {bend }}=-0.006 \mathrm{~N}$, tensile force $F_{\text {tens }}=10 \mathrm{~N}$ and pressure on the top surface $P=500 \mathrm{~Pa}$. The results are shown in Fig. 22.

The ratios of force load over the displacement were calculated, for the case of bending mode it is equal to $0.00006 \mathrm{~N} / \mathrm{mm}$ for the tensile case $0.24 \mathrm{~N} / \mathrm{mm}$, which means that compared to the optimised coated skin less bending and tensile forces are required to deform the optimised uncoated skin.

\subsection{Comparison between the optimised corrugated skins and trapezoidal corrugated skins}

In order to compare the optimised skins obtained in this work with the trapezoidal shape of corrugated skin, the models of coated and uncoated trapezoidal skins were simulated in ABAQYS as well. It was considered that ' $A$ ' and ' $C$ ' nodes are connected to create a trapezoidal shape, the rest of parameters such as geometry, thickness of materials, material properties were set the same as for corresponding optimised corrugated skins. The relationship between the applied force and displacement was examined for all cases and presented in Fig. 23.

As it can be seen from the graphs, that the optimised coated corrugated skin has slightly better bending stiffness than the coated corrugated trapezoidal skin, however the results for tensile stiffness are transverse. As for the uncoated corrugated skin the optimised one presents better tensile stiffness, but compromised bending stiffness than uncoated trapezoidal skin. However, as it was observed during the research it is always a trade-off between values of tensile and bending stiffness. 


\section{Conclusion}

In order to find an optimised shape of composite corrugated morphing skin, a Finite Element code was created in MATLAB to calculate the equivalent tensile stiffness, flexural stiffness and mass of the corrugated core. The aggregate and genetic algorithm optimisation methods were used to perform the multi-objective optimisation for different boundary conditions of the input nodes coordinates. The skin was optimised by three objectives and then the best configurations were selected as the best compromise points of the normalised Pareto surfaces.

Following the optimisation methods as explored and the results thus achieved in the paper above, it can be concluded that randomness of GA method does not provide a certain pattern of the shape, also the shapes obtained using this method are less smooth compared to results obtained with aggregate method. The fixation of thickness of materials during optimisation process improved the objective values for the case of uncoated corrugated skin, however degrade the results for coated corrugated skin. For all cases of coated and uncoated corrugated core with unfixed and fixed thickness of materials the best shape was obtained by means of four subdomains method. All optimised shapes were presented and comparative analysis of objective values was carried out. Where it was revealed that fluctuation range of mass and $1 / E I_{e q}$ values for the cases of fixed thickness is narrower than for the cases of fixed thickness of materials, however more wide for $E A_{e q}$. The ratio of thickness of corrugated core to thickness of elastomer coating in case of coated skin varies from 0.44 to 0.56, whereas for the best case it is equal to 0.56. Optimising the skin without elastomer coating doubles the thickness of corrugated core.

It was also revealed that for new optimised shapes of the skin the ratio of $E A_{e q} / E I_{e q}=0.04319$ for the case of coated core and equal to 0.095 in case of uncoated corrugated core. Furthermore, it was observed that the thickness of new optimised coated corrugated skin is 2.12 times thicker than the trapezoidal coated corrugated core obtained in paper [9]. This will lead to better bending stiffness to resist the aerodynamic pressure and buckling forces of morphing actuator. Also, the greater height of the skin decreases tensile stiffness of the panel that leads to a smaller actuator force needed for the deformation of the wing. 
The application of two different configurations: coated and uncoated corrugated skins were discussed. The comparison of the new optimised corrugated skin and trapezoidal corrugated skin was presented, where it was revealed that the coated optimised corrugated skin has slightly better bending stiffness than the coated corrugated trapezoidal skin, however the results for tensile stiffness are transverse. As for the uncoated corrugated skin the optimised one presents better tensile stiffness, but compromised bending stiffness than uncoated corrugated trapezoidal skin.

\section{References}

1. Abzug MJ, Larrabee EE. Airplane Stability and Control: A History of the Technologies That Made Aviation Possible. Cambridge: Cambridge University Press, 2002.

2. Raymer, D. Aircraft Design: A Conceptual Approach. Reston: American Institute of Aeronautics and Astronautics, 2006.

3. Barbarino S, Bilgen O, Ajaj RM, et al. A review of morphing aircraft. J of Intell Mater Syst and Struct 2011; 22(9): 823-877.

4. Chopra I. Status of application of smart structures technology to rotorcraft systems. J of the Am Helicopter Soc 2000; 45(4): 228-252.

5. Giurgiutiu V. Recent advances in smart-material rotor control actuation. In: 41st AIAA structures, structural dynamics and materials conference, Atlanta, GA, April, 2000.

6. Woods BKS, Fincham JHS, Friswell MI. Aerodynamic Modelling of the Fish Bone Active Camber Morphing Concept. Presented at the RAeS Applied Aerodynamic Conference. Bristol, July, 2014. 
7. Roosen D, Juhl B. Fatigue behaviour of sandwich foam core materials-comparison of different core materials. J of Adv Mater 2005; 37(2): 16.

8. Woods BKS, Friswell MI. Preliminary Investigation of a Fishbone Active Camber Concept. Proceeding of the ASME 2012 Conference on Smart Materials, Adaptive Structures and Intelligent Systems. Stone Mountain, September, 2012.

9. Dayyani I, Khodaparast HH, Woods BKS, Friswell MI. The design of a coated composite corrugated skin for the camber morphing airfoil. J of Intell Mater Syst and Struct 2014; 48(13):1-17

10. Yokozeki T, Takeda SI, Ogasawara T, et al. Mechanical properties of corrugated composites for candidate material of flexible wing structures. Composites Part A: Appl Sci and Manuf 2006; 37(10):1578-1586.

11. Dayyani I, Ziaei-Rad S, Friswell MI. The mechanical behavior of composite corrugated core coated with elastomer for morphing skins. J of Composite Mater 2013; 48(13):1623-1636

12. Gilchrist AC, Suhling JC, Urbanik TJ. Nonlinear finite element modelling of corrugated board. Applied Mechanics Division of the American Society of Mechanical Engineers 1998; 231: 101-106.

13. Luo S, Suhling JC, Considine JM, et al. (1992) The bending stiffnesses of corrugated board. In: Mechanics of Cellulosic Materials of the ASME (ed RW Perkins), ASME AMD- $16 \mathrm{~J}$ of Intell Mater Syst and Struct 1992; 145: 15-26, ASME winter annual meeting, Anaheim, CA, 8-13 November

14. Dayyani I, Woods BKS, Friswell MI, Khodaparast HH. The Optimal Design of a Coated Corrugated Skin for the FishBAC Morphing Wing. Presented at International Conference on Adaptive Structures and Technologies. Aruba, October, 2013.

15. Xia Y, Friswell MI, Saavedra Flores El. Equivalent models of corrugated panels. Int J of Solids and Struct 2012; 49:1453-1462 
16. Matlab. Fmincon. [online] available from <http://uk.mathworks.com/help/optim/ug/fmincon.html > [17 August 2016]

17. Dayyani I, Friswell MI. Multi-Objective Optimisation of Corrugated Morphing Skins. Manuscript submitted for publication, 2015.

18. Matlab. Genetic Algorithm Options. [online] available from <http://uk.mathworks.com/help/gads/examples/genetic-algorithm-options.html > [10 July 2016] 
Figures and tables

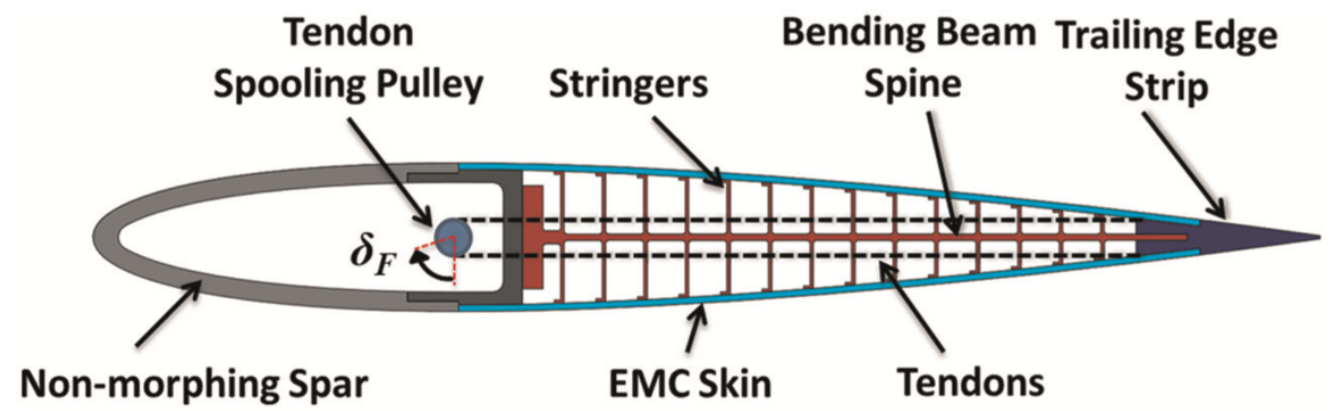

Figure 1 Fish Bone Active Camber concept [8]

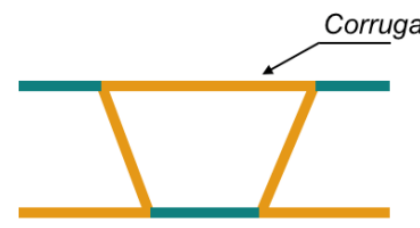

Reentrant

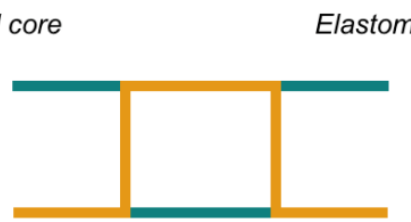

Rectangular

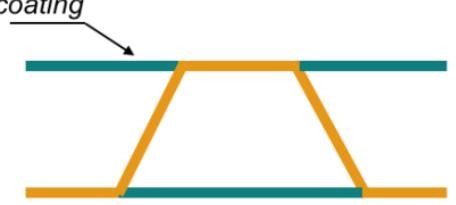

Trapezoidal

Figure 2 Three typical shapes of corrugation

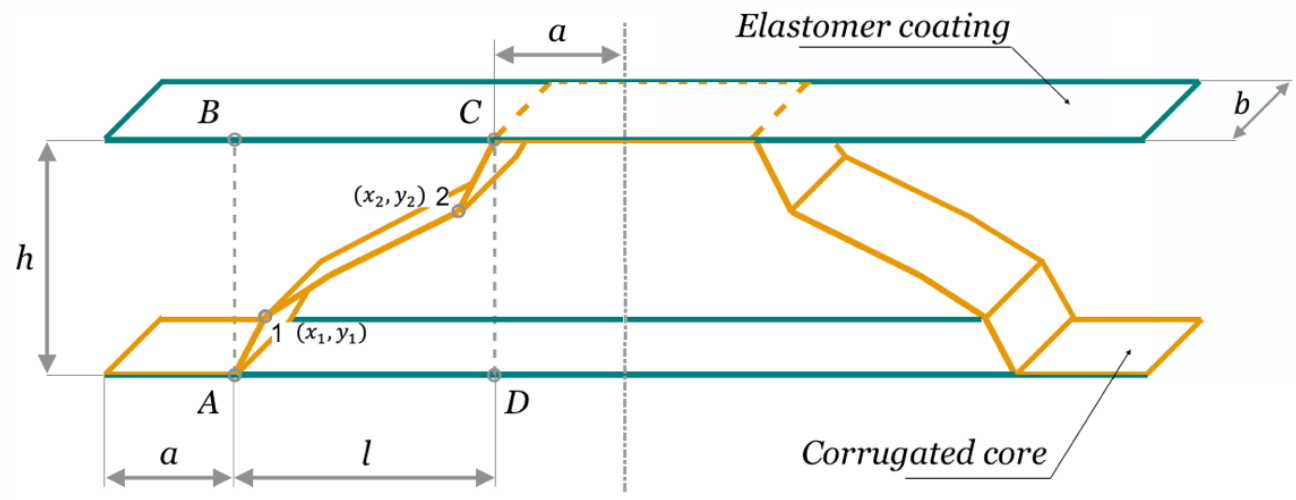

Figure $3 \mathrm{~A}$ unit cell of corrugation with arbitrary shape

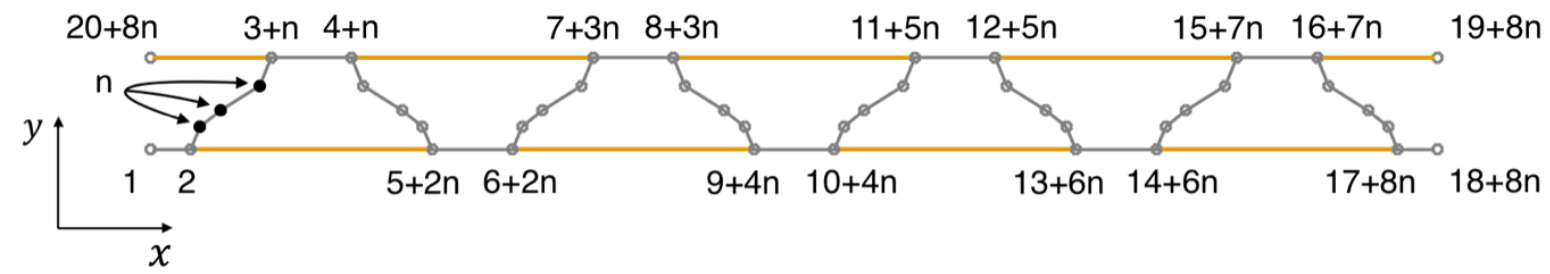

Figure 4 Discretization of the corrugated structure and set of independent nodes 

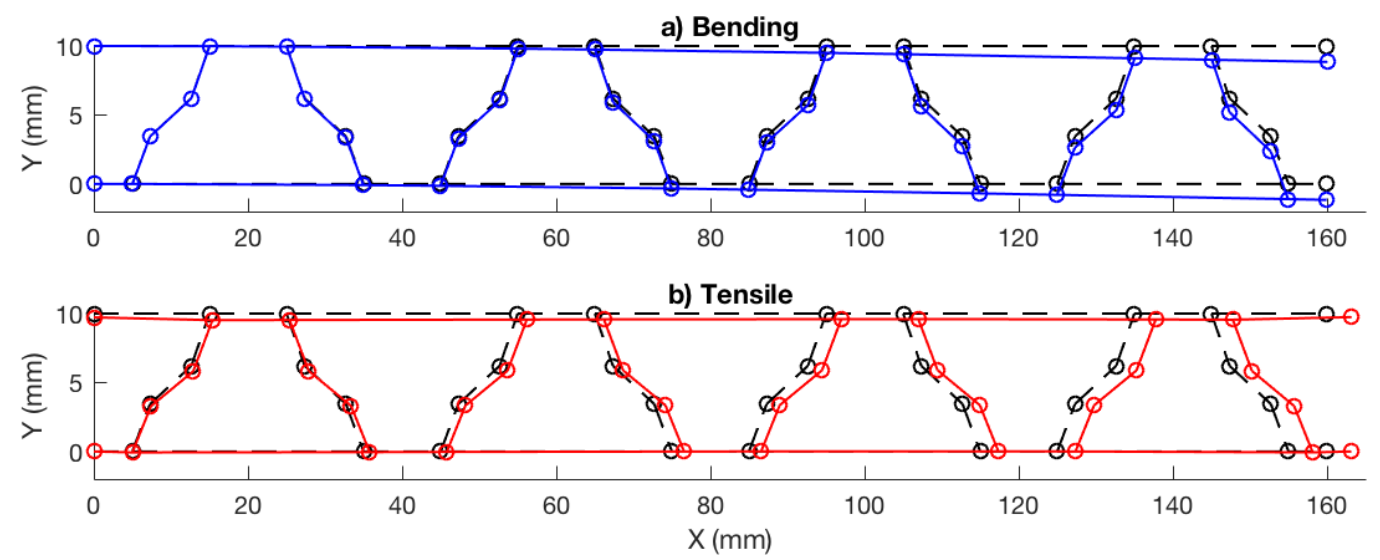

Figure 5 Undeformed and deformed shapes of the structure subjected to a) bending force $b$ ) tensile force

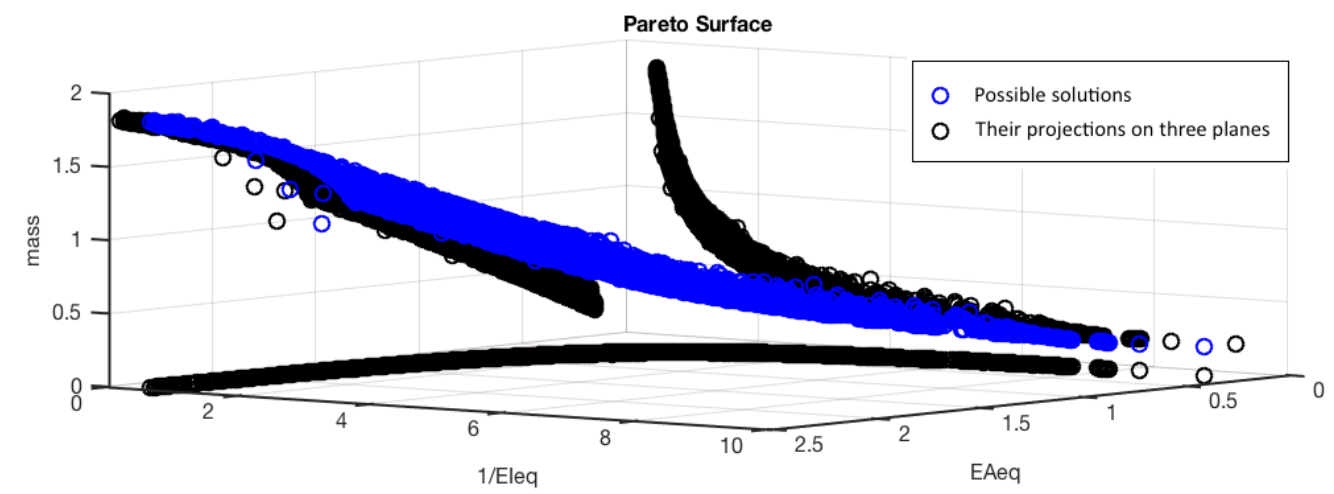

Figure 6 Pareto surface, multi-objective optimisation of corrugated skin (Aggregate optimisation method)

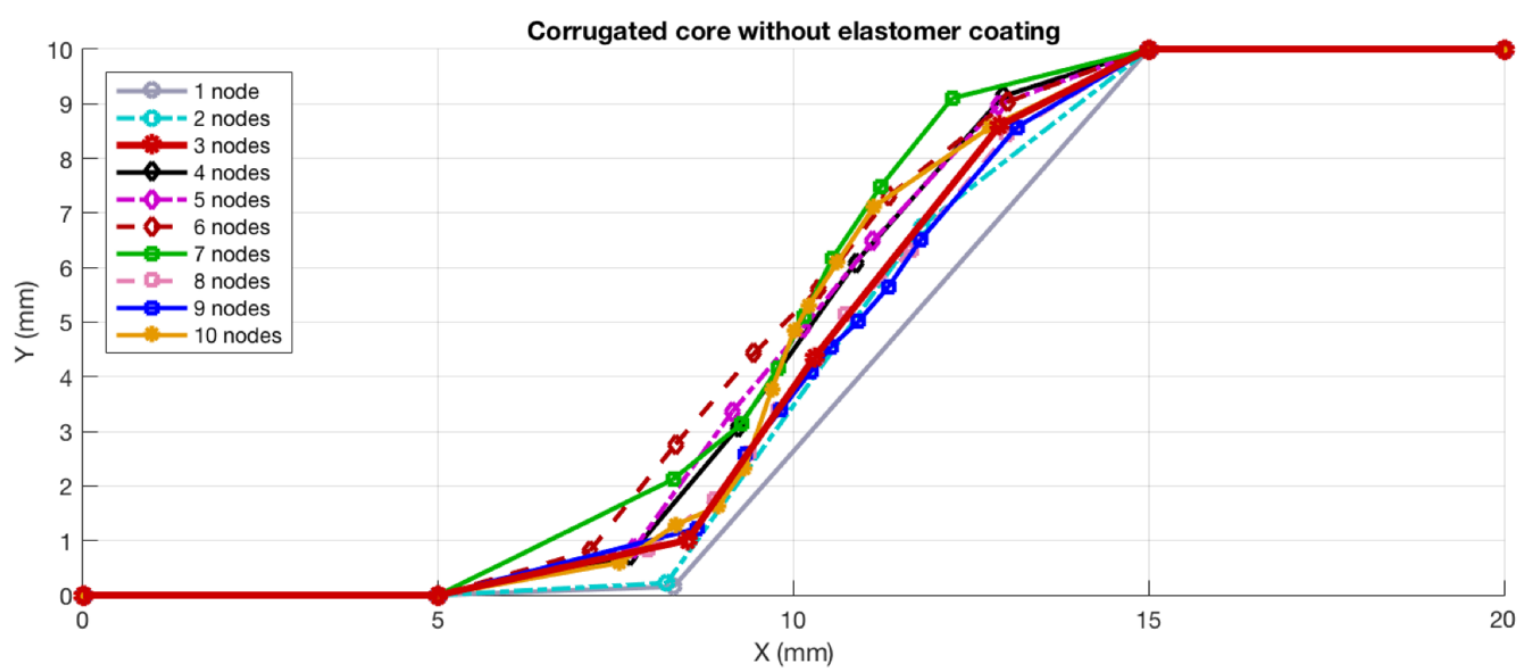

Figure 7 Optimised corrugation shapes for uncoated core obtained with different number of nodes 


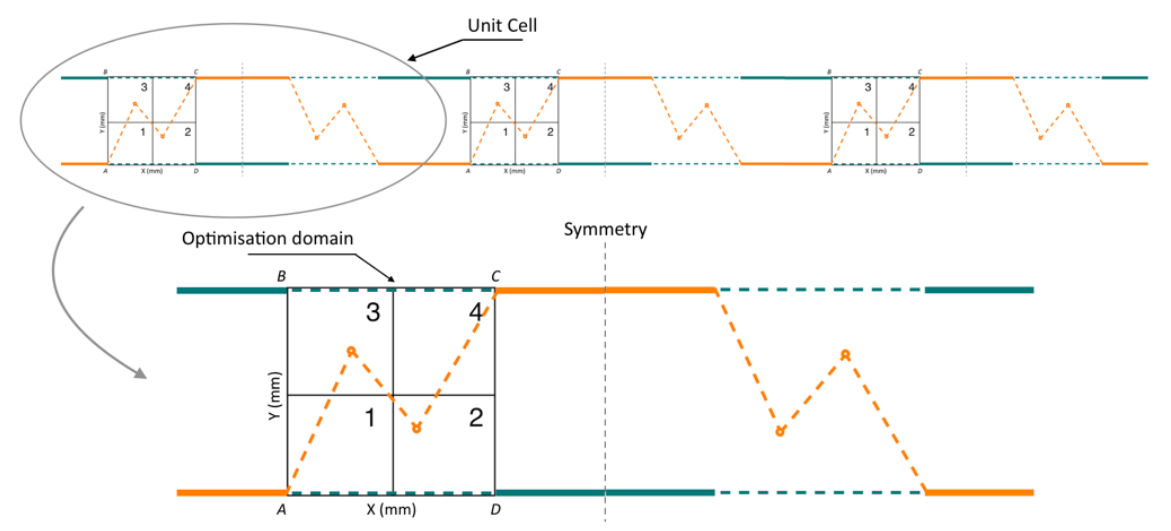

Figure 8 Unit cell with divided into four subdomains optimisation domain

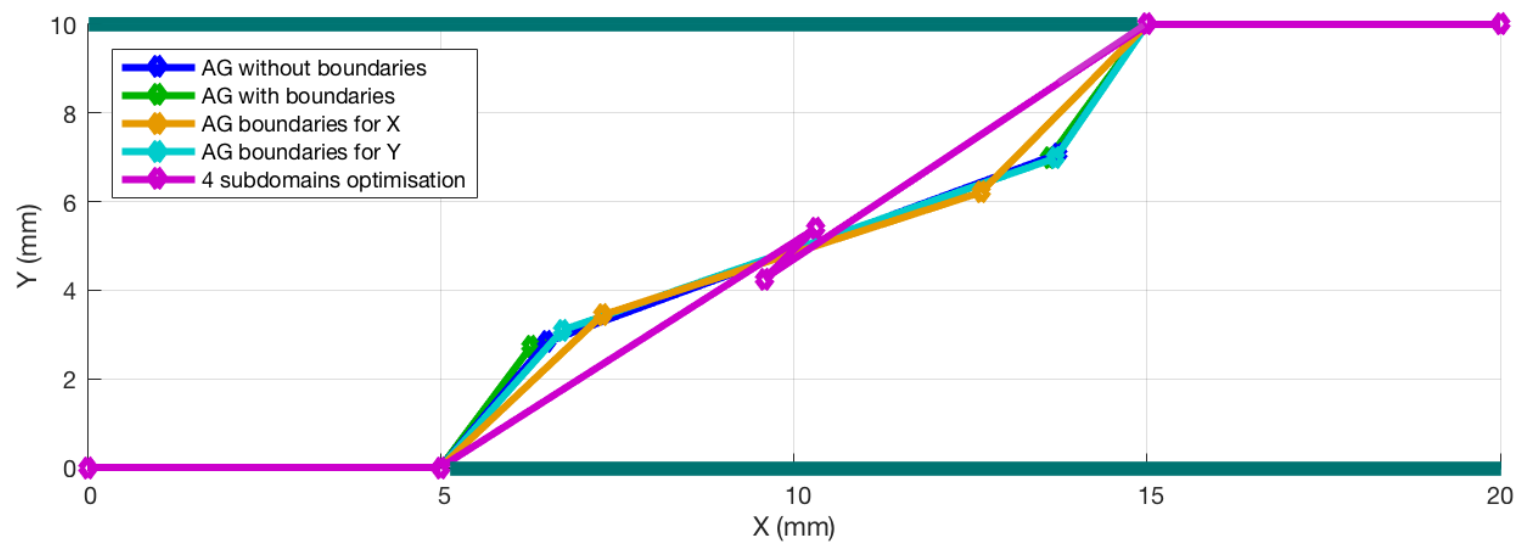

Figure 9 Optimised corrugation shapes for the case of coated corrugated core with unfixed thickness of materials
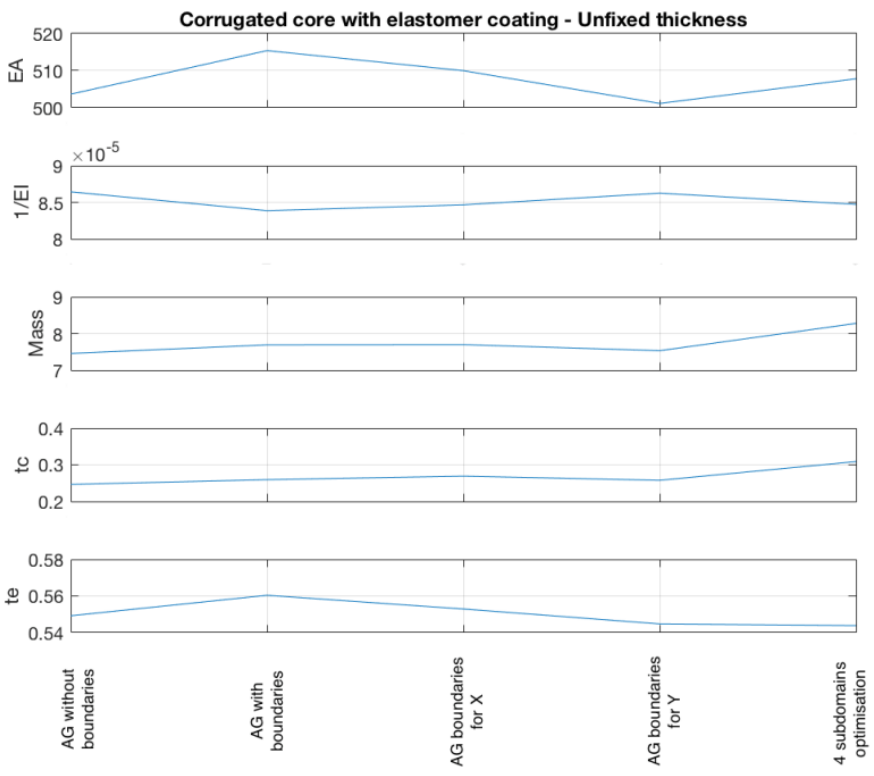

Figure 10 Comparative analysis of objective values and thickness of materials for different optimisation approaches 


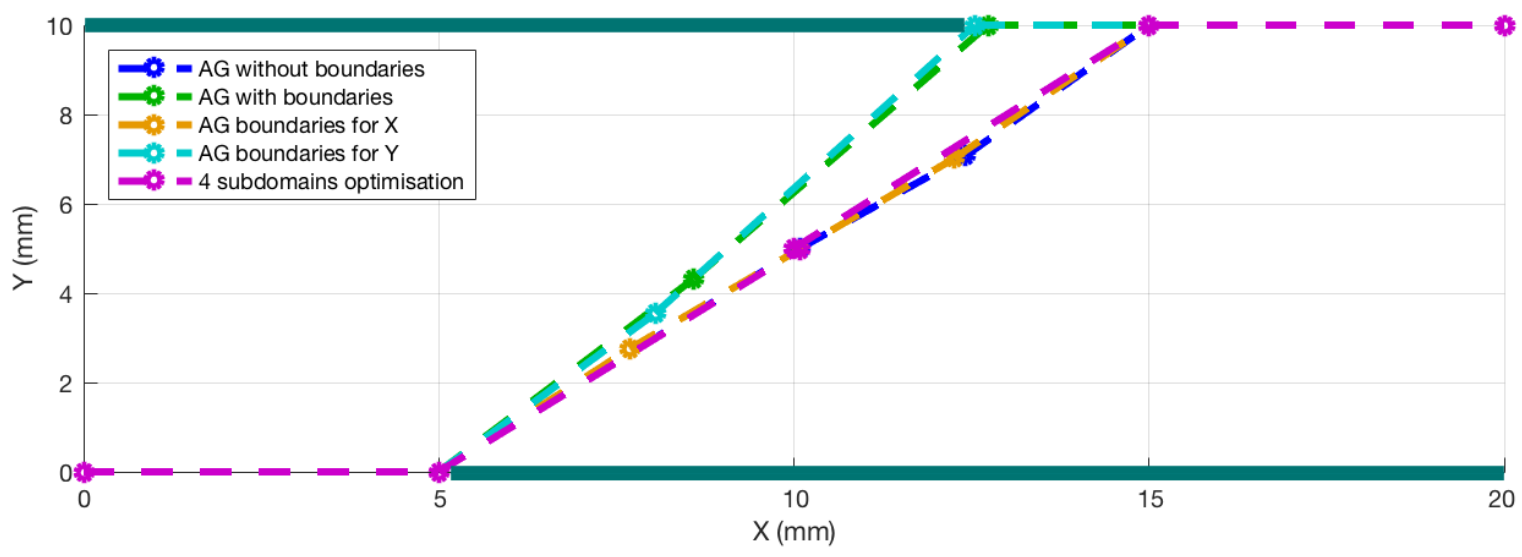

Figure 11 Optimised corrugation shapes for the case of coated corrugated core with fixed thickness of materials
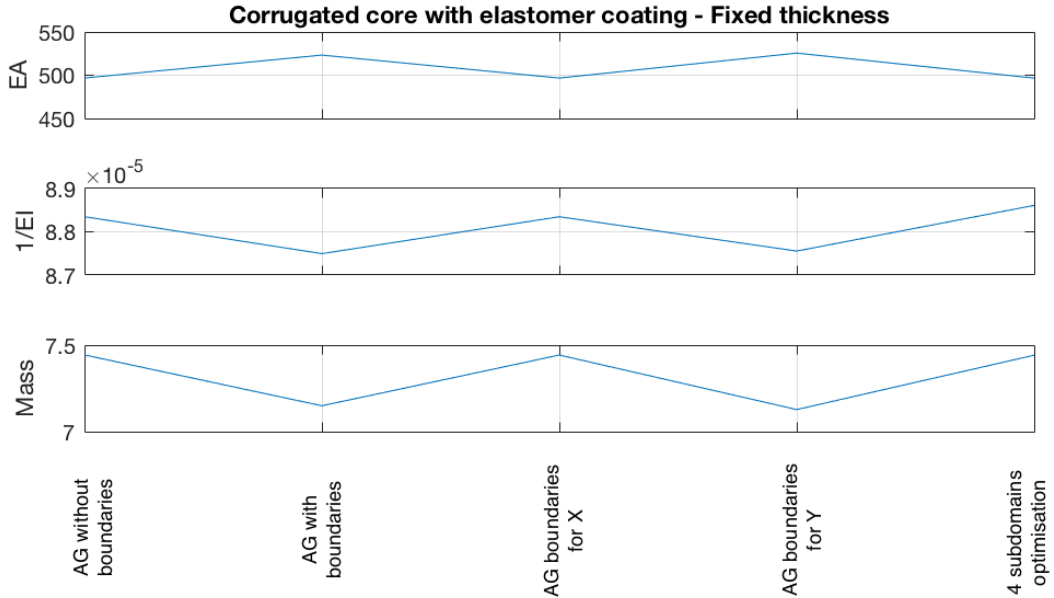

Figure 12 Comparative analysis of objective values for different optimisation approaches

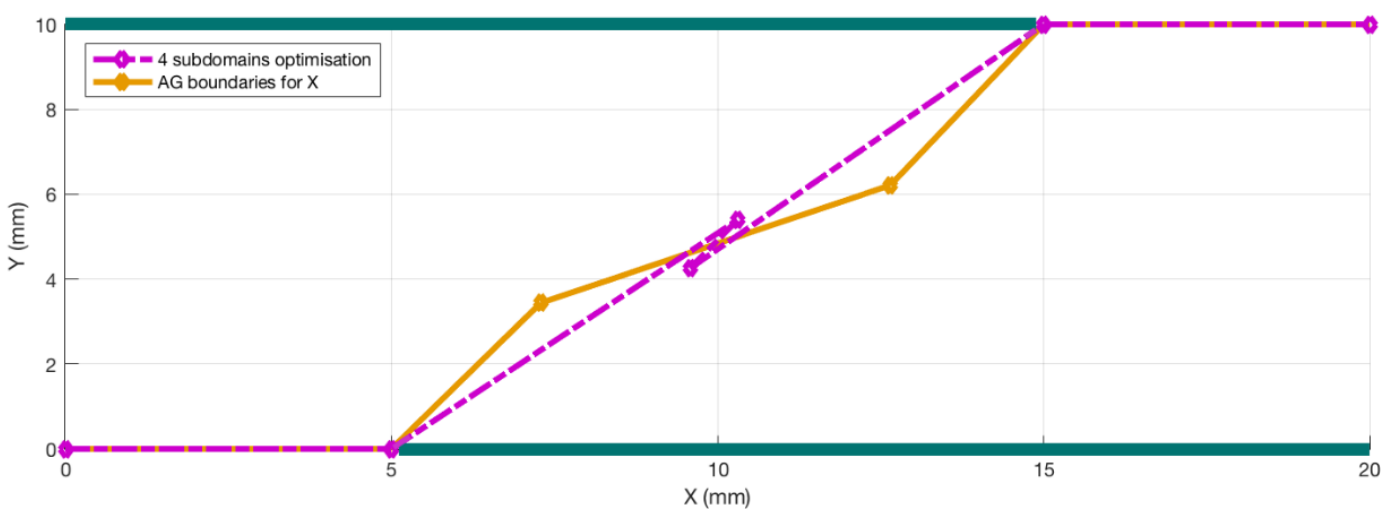

Figure 13 The best shapes obtained for the case of coated corrugated core

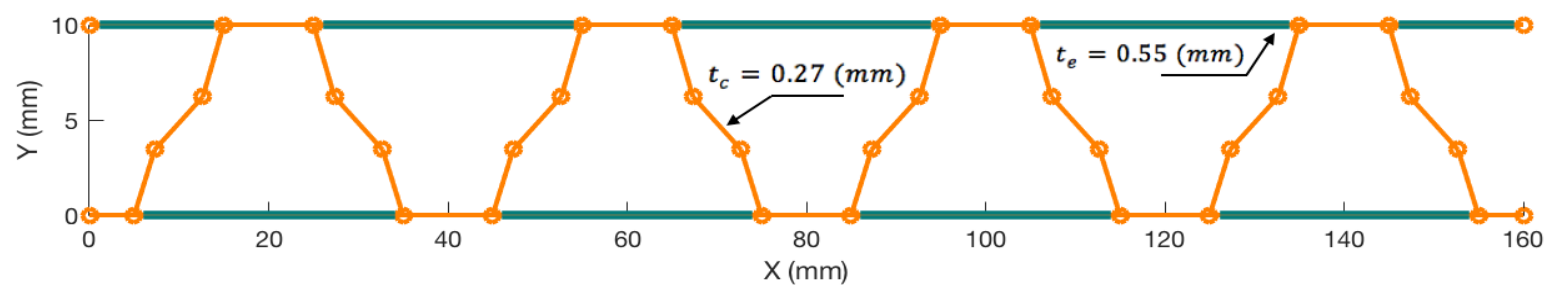

Figure 14 Shape optimised structure of coated corrugated skin 
a)
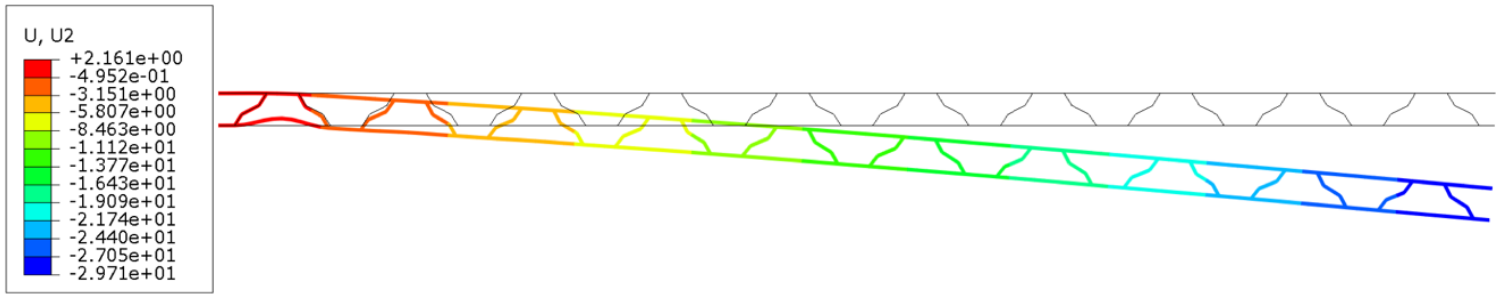

b)
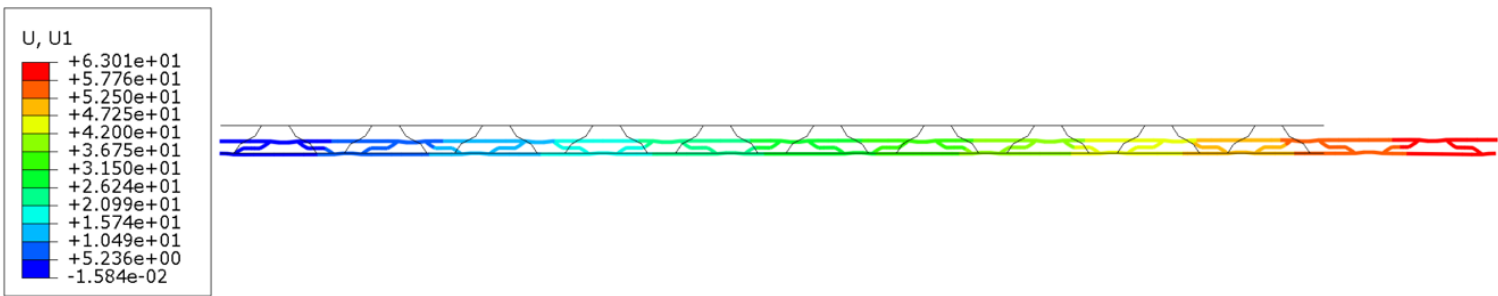

c)
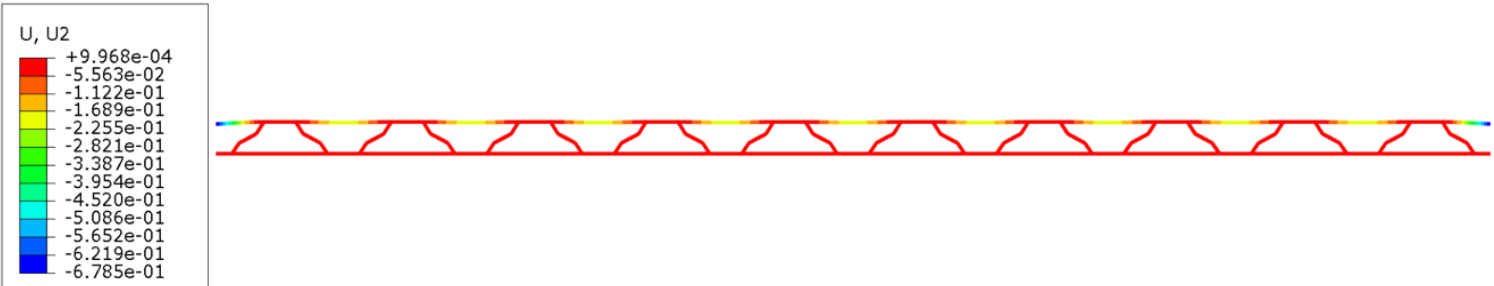

Figure 15 Deflection of the structure objected to a) bending, b) tensile, c) pressure load conditions

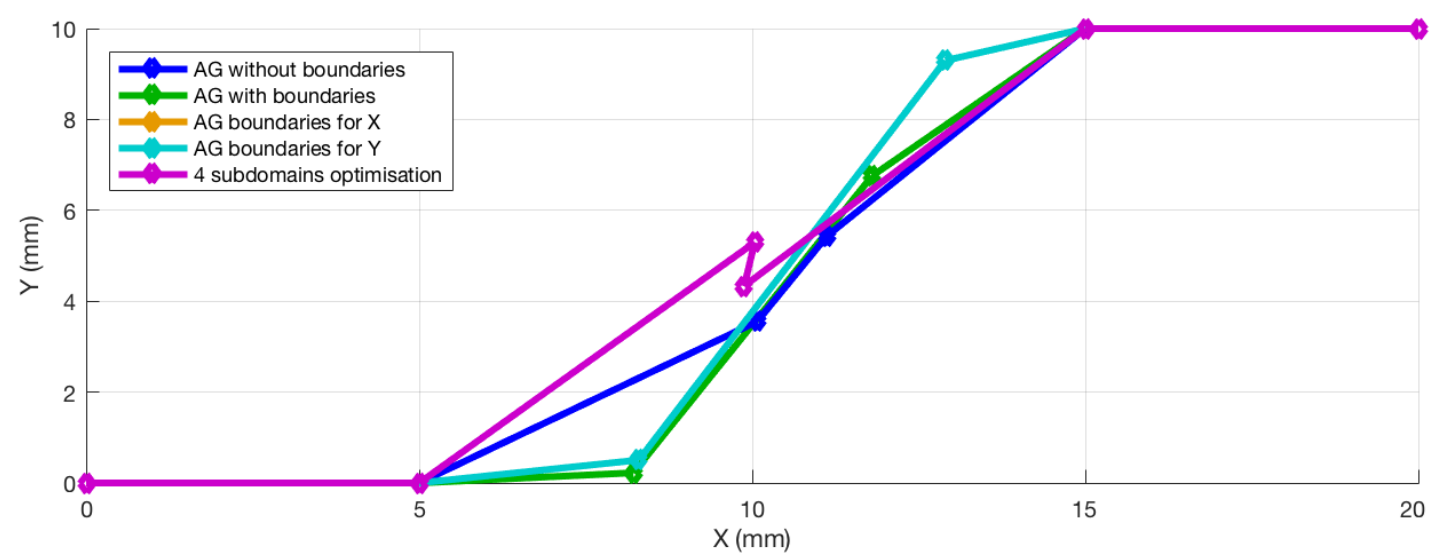

Figure 16 Optimised corrugation shapes for the case of uncoated corrugated core with unfixed thickness of material 

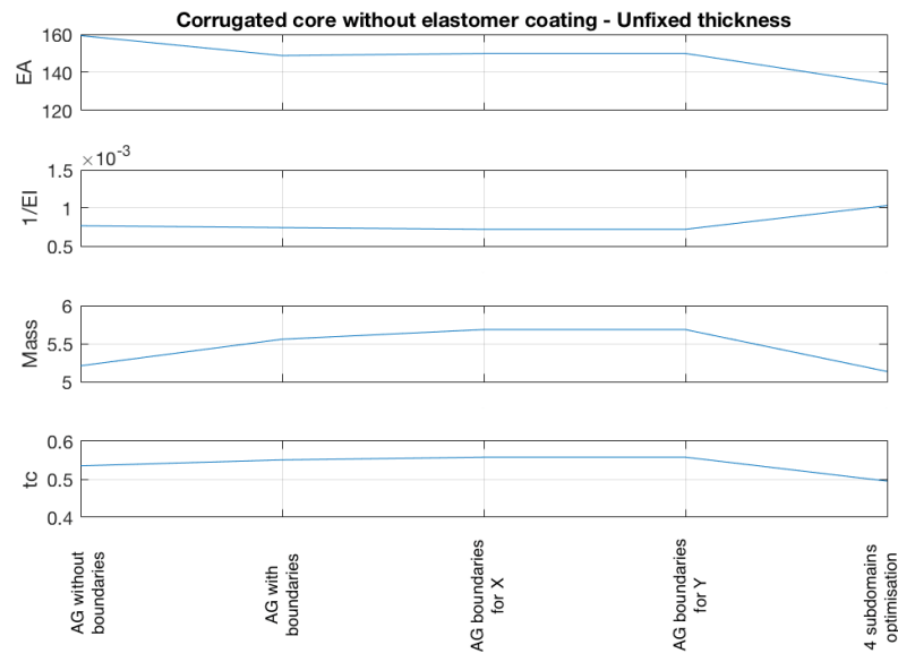

Figure 17 Comparative analysis of objective values and thickness of material for different optimisation approaches

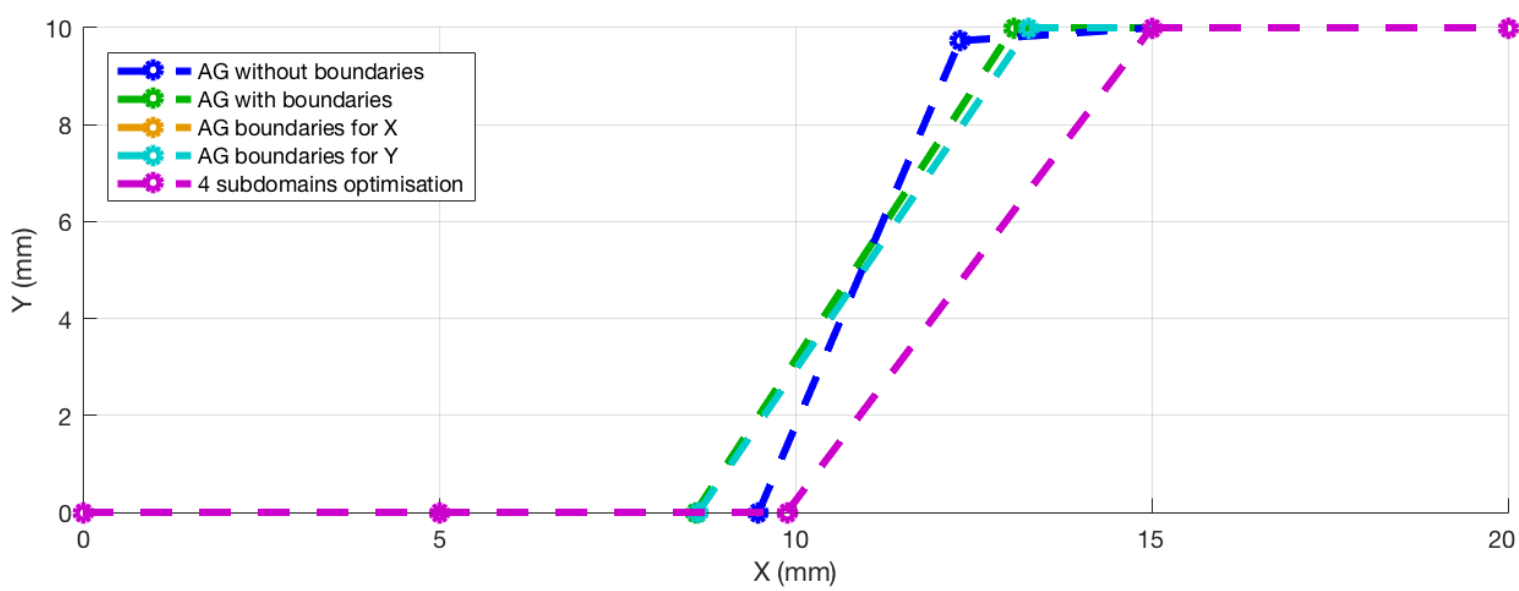

Figure 18 Optimised corrugation shapes for the case of uncoated corrugated core with fixed thickness of material
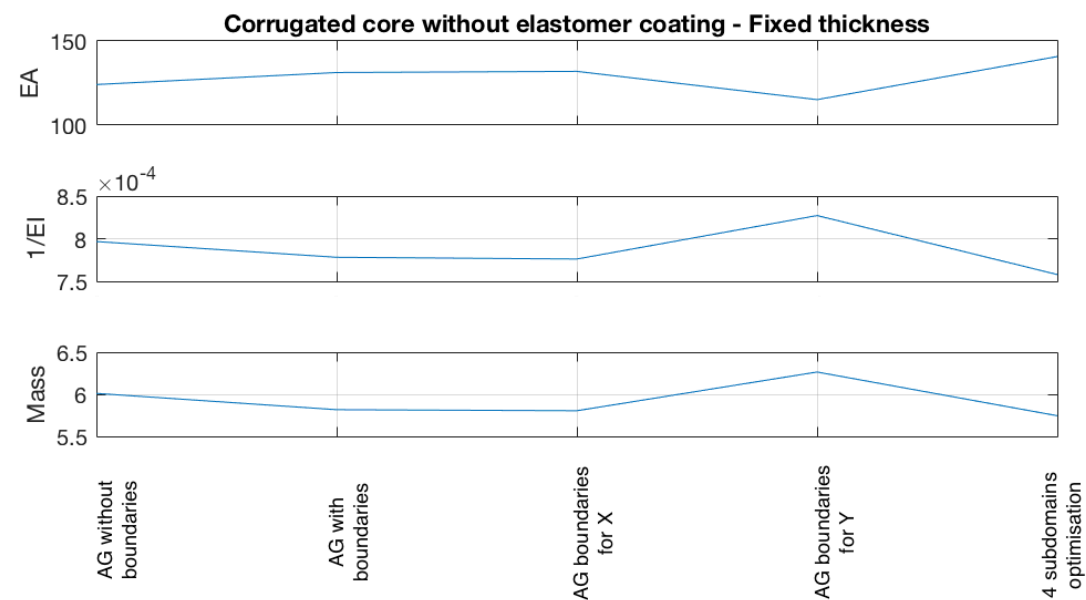

Figure 19 Comparative analysis of objective values for different optimisation approaches 


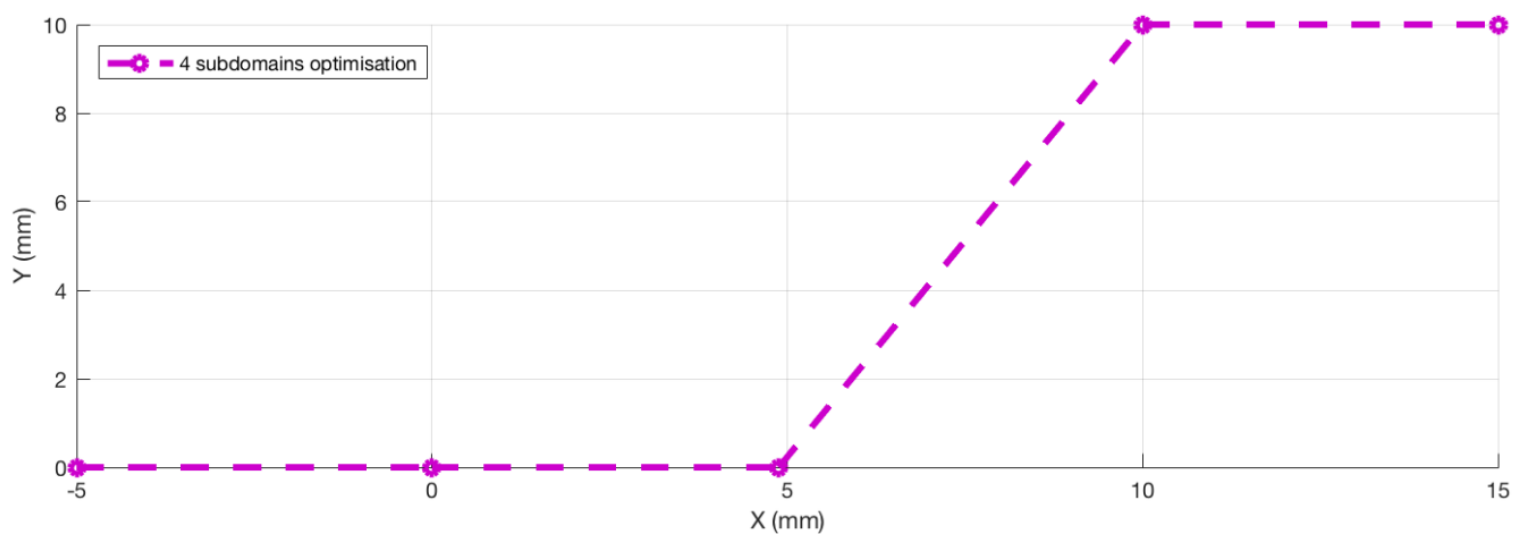

Figure $\mathbf{2 0}$ The best shape obtained for the case of uncoated corrugated core

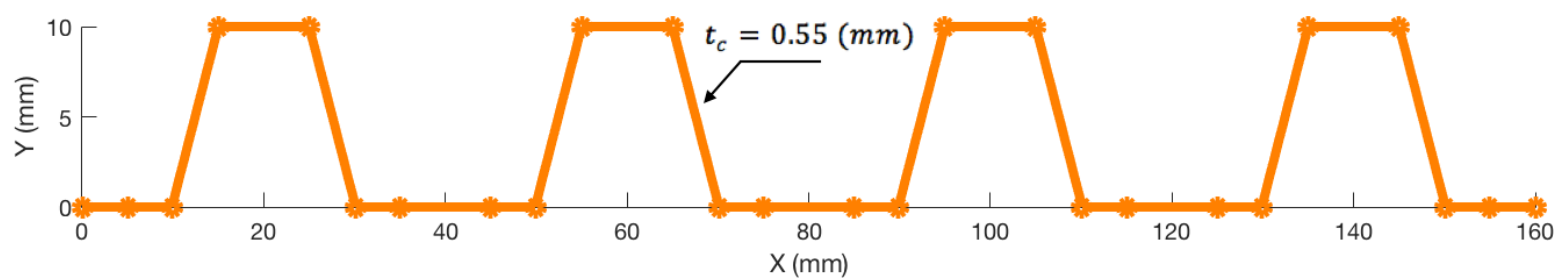

Figure 21 Shape optimised structure of uncoated corrugated skin

a)
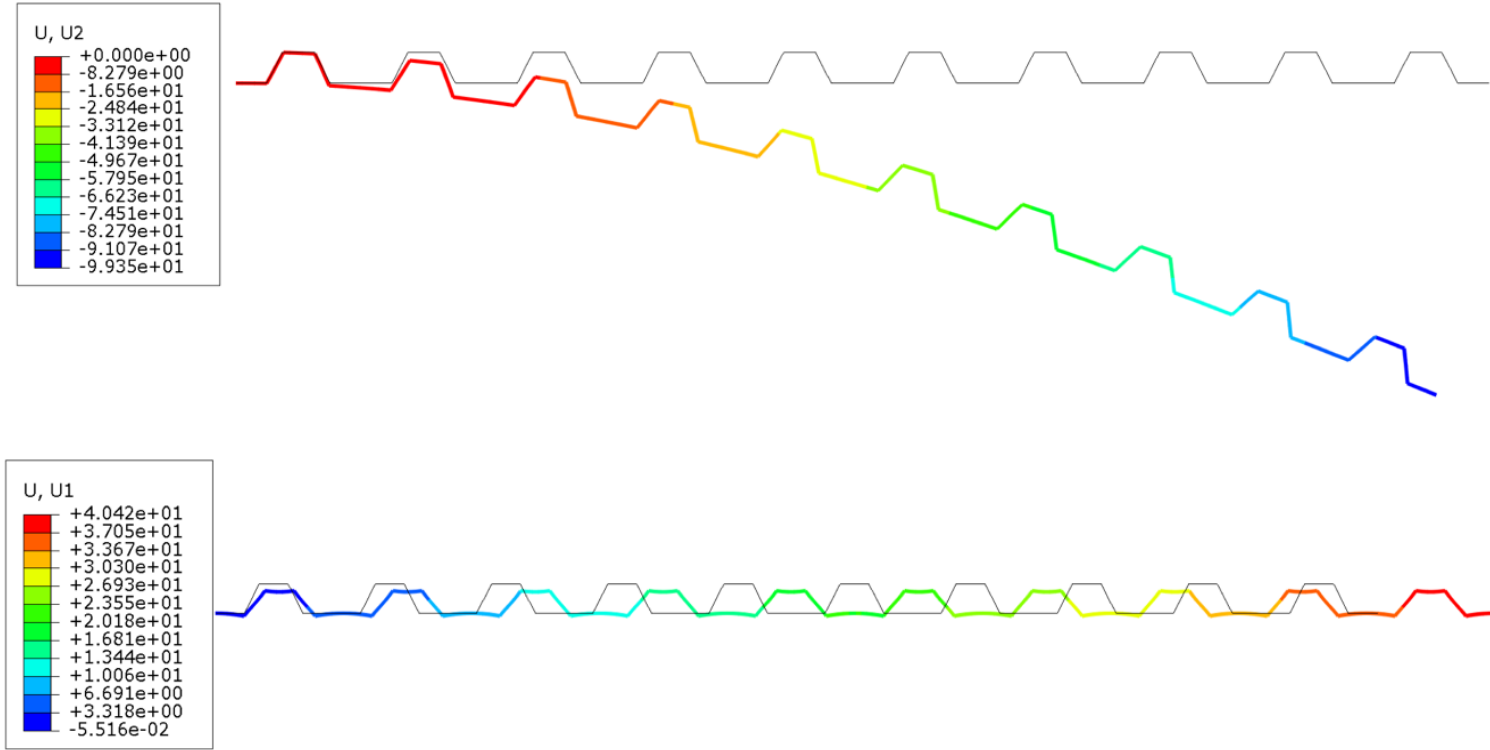

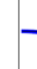
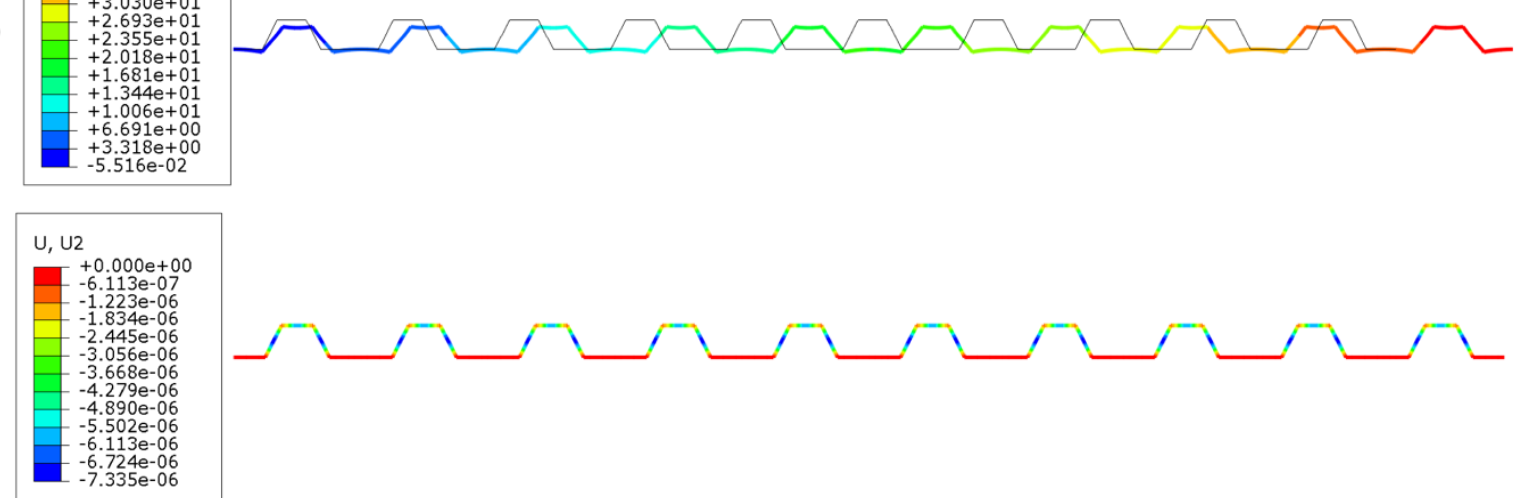

Figure 22 Deflection of the structure objected to a) bending, b) tensile, c) pressure load conditions 
a)

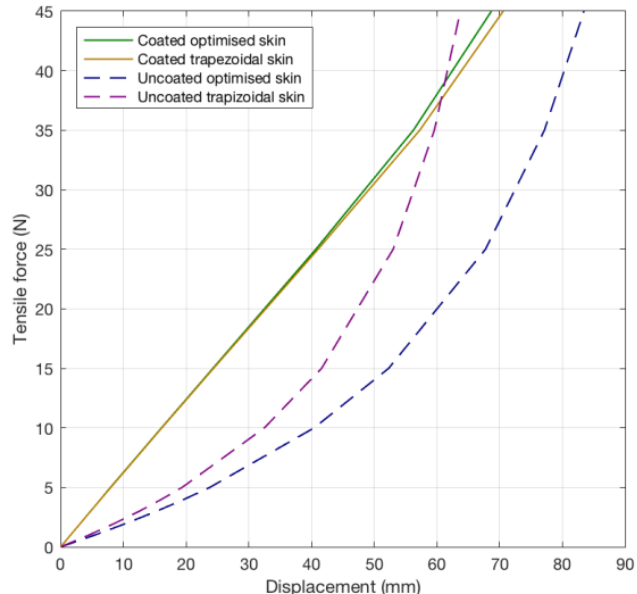

b)

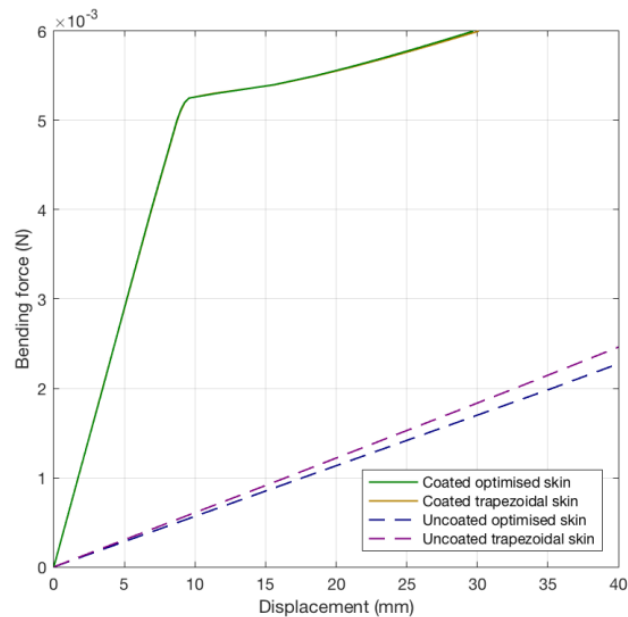

Figure 23 Force displacement curves for optimised corrugated skins and for corresponding trapezoidal corrugated skins: a) tensile and $b$ ) bending

\begin{tabular}{|c|l|l|}
\multicolumn{1}{|l}{ Variables } & Description & $5 \mathrm{~mm}$ \\
\hline$a$ & Geometrical component & $10 \mathrm{~mm}$ \\
$h$ & Height & $10 \mathrm{~mm}$ \\
$l$ & Length & $25 \mathrm{~mm}$ \\
$b$ & Width of panel & $0.002 \mathrm{~g} / \mathrm{mm}^{3}$ \\
$\rho_{c}$ & Density of composite corrugated core & $0.001522 \mathrm{~g} / \mathrm{mm}^{3}$ \\
$\rho_{e}$ & Density of elastomer coating & $4.5 \mathrm{GPa}$ \\
$E_{c}$ & Young's modulus of composite corrugated core & $13.5 \mathrm{MPa}$ \\
$E_{e}$ & Young's modulus of elastomer coating &
\end{tabular}

Table 1 Fixed values for the geometry and material properties of the corrugation unit cell

\begin{tabular}{|l|l|} 
Description & 4 \\
\hline Number of unit cells & $25 \mathrm{~mm}$ \\
Width of panel & $0.002 \mathrm{~g} / \mathrm{mm}^{3}$ \\
Density of composite corrugated core & $0.001522 \mathrm{~g} / \mathrm{mm}^{3}$ \\
Density of elastomer coating & $4.5 \mathrm{GPa}$ \\
Young's modulus of composite corrugated core & $13.5 \mathrm{MPa}$ \\
Young's modulus of elastomer coating & 0.225 \\
Poisson's Ratio of corrugated core & 0.3 \\
\hline
\end{tabular}

Table 2 Fixed geometric parameters and material properties of the skin for ABAQUS simulation 


\begin{tabular}{|c|ccc|ccc|}
\hline \multirow{2}{*}{$\begin{array}{c}\text { Node } \\
\text { (refer to Fig. 4) }\end{array}$} & \multicolumn{3}{|c|}{ Prescribed DOFs } & \multicolumn{3}{c|}{ Bending modelling } \\
\cline { 2 - 7 } & \multicolumn{2}{|c|}{ Tensile modelling } & $\theta_{z}$ & $U_{x}$ & $U_{y}$ & $\theta_{z}$ \\
\hline 1 & $U_{x}$ & $U_{y}$ & 0 & 0 & 0 & 0 \\
$18+8 \mathrm{n}$ & 0 & 0 & 0 & Free & Free & Free \\
$19+8 \mathrm{n}$ & Free & 0 & 0 & Free & Free & Free \\
$20+8 \mathrm{n}$ & Free & Free & 0 & 0 & 0 & 0 \\
\hline
\end{tabular}

Table 3. Prescribed boundary conditions applied to the coated corrugated core in tensile and bending models

\begin{tabular}{|c|ccc|}
\hline Displacement & MATLAB & ABAQUS & Error \\
\hline Bending modelling & -1.1567 & -1.157 & $0.03 \%$ \\
\hline Tensile modelling & 3.1608 & 3.196 & $1.11 \%$ \\
\hline
\end{tabular}

Table 4 Validation of the FE equivalent code against ABAQUS

\begin{tabular}{cccccccc}
\hline$w_{1}$ & $w_{2}$ & $w_{3}$ & $t_{c}(m m)$ & $t_{e}(\boldsymbol{m m})$ & $E A_{e q}(\boldsymbol{N})$ & $1 / E I_{e q}\left(N_{m m}^{2}\right)^{-1}$ & $\operatorname{mass}(\mathrm{g})$ \\
0.34 & 0.39 & 0.27 & 0.2690 & 0.5529 & 509.9836 & 0.0000847 & 7.6984 \\
\hline
\end{tabular}

Table 5 Objective values, thickness of materials and weights distribution for the decision making configuration

\begin{tabular}{ccccccc}
\hline$w_{1}$ & $w_{2}$ & $w_{3}$ & $t_{c}(\boldsymbol{m m})$ & $\boldsymbol{E} A_{e q}(\boldsymbol{N})$ & $\mathbf{1} / \boldsymbol{E I}_{\text {eq }}\left(\boldsymbol{N m m}^{2}\right)^{-1}$ & $\boldsymbol{m a s s}(\mathrm{g})$ \\
0.69 & 0.03 & 0.28 & 0.55 & 115.1673 & 0.000827 & 6.2645 \\
\hline
\end{tabular}

Table 6 Objective values, thickness of materials and weights distribution for the best shape case 
2016-10-15

\title{
Shape optimisation of composite corrugated morphing skins
}

\author{
Ermakova, Anna
}

Elsevier

Anna Ermakova, Iman Dayyani, Shape optimisation of composite corrugated morphing skins, Composites Part B: Engineering, Volume 115, Issue April, 2017, pp. 87-101

http://dx.doi.org/10.1016/j.compositesb.2016.10.029

Downloaded from Cranfield Library Services E-Repository 